\title{
BMJ Open Comparison of prevalence and associated factors of depressive disorder between patients with head and neck cancer and those with lung cancer at a tertiary hospital in Taiwan: a cross- sectional study
}

Yu Lee, ${ }^{1}$ Chi-Fa Hung, ${ }^{1}$ Chih-Yen Chien, ${ }^{2}$ Pao-Yen Lin, ${ }^{1}$ Meng-Chih Lin, ${ }^{3}$ Chin-Chou Wang, ${ }^{3}$ Hung-I Lu, ${ }^{4}$ Yung-Che Chen, ${ }^{3}$ Mian-Yoon Chong, ${ }^{1}$ Liang-Jen Wang (i) ${ }^{5}$

To cite: Lee Y, Hung C-F, Chien C-Y, et al. Comparison of prevalence and associated factors of depressive disorder between patients with head and neck cancer and those with lung cancer at a tertiary hospital in Taiwan: a crosssectional study. BMJ Open 2020;10:e037918. doi:10.1136/ bmjopen-2020-037918

- Prepublication history and additional material for this paper are available online. To view these files, please visit the journal online (http://dx.doi org/10.1136/bmjopen-2020037918).

Received 22 February 2020 Revised 13 May 2020 Accepted 29 May 2020

Check for updates

(C) Author(s) (or their employer(s)) 2020. Re-use permitted under CC BY-NC. No commercial re-use. See rights and permissions. Published by BMJ.

For numbered affiliations see end of article.

Correspondence to Dr Liang-Jen Wang; wangliangjen@gmail.com

\section{ABSTRACT}

Objective Depression is a common comorbidity in cancer patients. This study aimed to compare the prevalence and associated factors of depressive disorder between patients with head and neck cancer (HNC) and those with lung cancer (LC).

Design This study used a cross-sectional design with consecutive sampling.

Setting A medical centre (Kaohsiung Chang Gung Memorial Hospital, Taiwan).

Participants Patients for the study were recruited from the HNC and LC outpatient clinic and inpatient ward from March 2016 to February 2018. Patients with HNC and LC were enrolled and assessed using the Mini International Neuropsychiatric Interview (MINI).

Primary and secondary outcome measures The primary outcome was psychiatric diagnoses assessed using the MINI. The secondary outcomes were psychological well-being assessed using the Beck Anxiety Inventory, Taiwanese Depression Questionnaire, Brief Fatigue Inventory, Numeric Pain Rating Scale and the List of Threatening Experiences Questionnaire.

Results In total, 113 HNC patients and 104 LC patients were recruited for the study. The most common psychiatric comorbidity of HNC patients was alcohol use disorder (49.6\%), followed by adjustment disorder (20.4\%) and depressive disorder (11.5\%). The most common psychiatric comorbidity of LC patients was depressive disorder (25.0\%), followed by adjustment disorder (17.3\%), alcohol use disorder (3.8\%) and insomnia disorder (3.8\%). Among HNC patients, a self-harm history was positively associated with depression ( $\mathrm{OR}=11.91 ; 95 \% \mathrm{Cl}, 1.47$ to 96.83), and a higher educational level was negatively associated with depression $(0 \mathrm{R}=0.77 ; 95 \% \mathrm{Cl}, 0.66$ to 0.91). Among LC patients, severity of stressor ( $\mathrm{OR}=2.78$; $95 \% \mathrm{Cl}, 1.50$ to 5.15$)$ and severity of anxiety ( $\mathrm{OR}=1.18$; $95 \% \mathrm{Cl}, 1.04$ to 1.34 ) were two significant factors associated with depression.

Conclusion We reported the prevalence and associated factors of depression between patients with HNC
Strengths and limitations of this study

We carried out a comprehensive study that compared depression and associated factors between patients with head and neck cancer and those with lung cancer.

- The high response rate and the use of a structured clinical interview by psychiatrists are two strengths of this study.

- The small sample size in our study limits the statistical power of the findings.

- We used a cross-sectional design; therefore, no causal relationships could be identified.

and those with LC. Clinicians should be aware of this comorbidity and the associated risk factors, and conduct intervention programmes to prevent these cancer patients from developing depression.

\section{INTRODUCTION}

Head and neck cancer (HNC) is the seventh most common cancer and the ninth most frequent cause of death from cancer worldwide, with an estimated more than 550000 occurrences and 300000 deaths annually. ${ }^{1}$ Lung cancer (LC) is the leading cause of cancer mortality around the world. ${ }^{2}$ In the US, LC had the second highest incidence of all cancers, and official data reported a total of 83550 males and 70500 females died from LC in the US in 2018. ${ }^{3}$ The overall 5-year survival rate of patients with $\mathrm{HNC}$, around $40 \%$ to $50 \%$, is one of the lowest among all cancers and has not significantly changed during the last two decades. ${ }^{4}$ The overall 5 -year survival rate of $\mathrm{LC}$ varies globally, but is consistently low due to late-stage detection. ${ }^{5}$ 
Early LC and HNC detection for the sake of mortality reduction has become an important public health issue.

Depressive disorders include major depressive disorder (MDD), persistent depressive disorder, other specified depressive disorder, premenstrual dysphoric disorder and disruptive mood dysregulation disorder based on Diagnostic and Statistical Manual of Mental Disorders, Fifth Edition, (DSM-5) classification. ${ }^{6}$ MDD, a common comorbidity in cancer patients, involves depressed mood, decreased interest, poor appetite, insomnia, cognitive impairment, pessimistic thinking or even suicidal ideas/attempts. ${ }^{7} 8$ Depression significantly increases cancer patients' risk of noncompliance with treatment, and contributes to a poorer treatment response and increased rates of hospital admission; worst of all, it can lead to mortality and even suicide. ${ }^{9-13}$ Both HNC and LC are known to be associated with an increased risk of depressive disorder. ${ }^{14-16}$ Prior studies revealed that the incidence of depressive disorder in HNC patients was one of the highest among all cancer patients, with estimated prevalence rates ranging from $19 \%$ to $57 \%$, and with a $25 \%$ occurrence rate prior to cancer treatment. ${ }^{17}$ The discrepancy in rates is mainly due to differences in (1) method of investigation, (2) measurement, (3) samples, (4) follow-up period, and (5) degree of severity or staging of cancer. ${ }^{14}$ In general, a higher rate of depression is estimated when using patient self-reported questionnaires than when there are structured interviews conducted by experienced psychiatrists. ${ }^{14}$

Previous studies of LC patients showed high rates of clinically significant depressive disorder shortly after diagnosis $(21 \%$ to $44 \%),{ }^{18}$ and after treatment completion (29\% to $44 \%) .{ }^{19}$ A review paper from Massie found that the prevalence of depression in LC patients in prior studies ranged from $11 \%$ to $44 \% .{ }^{20}$ Higher rates of depression were estimated when using patient selfreported questionnaires; therefore, overrating might be seen in most items of somatic symptoms, particularly in patients suffering from physical illness. ${ }^{21}$ It is important that structured diagnostic interviews by a senior psychiatrist are used to accurately detect psychiatric diagnoses of depressive disorder in cancer patients.

Understanding the risk factors of depression may be helpful in developing strategies for preventing depression among cancer patients. Literature reviews revealed that depression was more prevalent in HNC patients with the following characteristics: female, younger, having a previous history of depression, alcohol use, during the initial treatment, during advanced stages of cancer and among those with poor family support. ${ }^{22}$ Previous studies have shown that the risk factors for depressive disorder in patients with LC include cancer stage ${ }^{23}$ health behaviours such as alcohol intake ${ }^{24}$ or smoking ${ }^{25}$; physical symptoms such as pain $^{26}$ or fatigue ${ }^{27}$; and personal characteristics such as age, ${ }^{27}$ sex (male), ${ }^{28}$ employment status ${ }^{29}$ or lower educational level. ${ }^{30}$ There is a paucity of studies comparing associated factors between patients with HNC and those with LC.
Taken together, LC and HNC are two of the most prevalent cancers comorbid with depression. LC is the leading cause of cancer mortality, and HNC is the fifth most common cause of cancer mortality in Taiwan 2018. ${ }^{31}$ Many oncologists see both LC and HNC patients in their clinical practice simultaneously. Moreover, the cancers in HNC and LC patients are commonly smoking-related, which warrants clarifying the differences between these two cancers in terms of depression and its associated factors. Therefore, this study aimed to compare the prevalence and associated factors of depressive disorder between patients with HNC and those with LC.

\section{METHODS \\ Participants}

All procedures performed in studies involving human participants were in accordance with the Declaration of Helsinki (1964) and its later amendments or comparable ethical standards. The Institutional Review Board at Chang Gung Memorial Hospital has approved this study (IRB No. 104-7000B).

According to previous epidemiological studies, comorbidity rates of depression among patients with $\mathrm{HNC}$ and those with LC are about $10 \%$ to $20 \%$ and $20 \%$ to $30 \%$, respectively. With regard to the predictors of depression, the individual contribution of each factor may be modest. Given an effect size of 0.4 , an alpha value of 0.05 , power (1-Beta) $=0.8$, and three repeated measurements, a correlation of 0.5 to 0.6 and a non-sphericity correction of 1100 patients in each cancer group were needed to achieve enough statistical power to detect depressionassociated factors. The above statistical power calculation used the Gpower V.3.17. ${ }^{32}$

This study used a cross-sectional design with consecutive sampling. Participants were recruited from the ear, nose and throat, chest and cardiovascular surgery outpatient/inpatient departments at a tertiary hospital from March 2016 to February 2018. This hospital has 2754 beds and provides services to 5000 cancer patients per year in southern Taiwan. Inclusion criteria were as follows: (1) patients with newly diagnosed HNC or LC; and (2) patients with the ability to understand the study procedure and complete the questionnaires. Exclusion criteria were as follows: (1) patients with a diagnosis of schizophrenia, schizoaffective disorder or bipolar I disorder; (2) patients with dementia or severe cognitive impairment; (3) patients whose depression was caused by another general medical condition (eg, thyroid disease) or induced by substances; and (4) patients who were too weak to complete the questionnaire or clinical interview.

\section{Study procedures}

Study procedures were as follows: (1) newly-diagnosed patients visiting our collaborative care clinic or admitted to our ward were invited consecutively to take part in this study. Once our research assistant received a referral from the outpatient clinic or wards from in-charge doctors or 
case managers, our research assistant went to the above settings to contact the patients. After explaining the study procedure and aims, those who agreed to sign an informed consent form were enrolled in the study; (2) a senior psychiatrist (YL) made the psychiatric diagnoses using the Mini International Neuropsychiatric Interview (MINI) $;^{33}$ and (3) assessment tools, including the Brief Fatigue Inventory (BFI) ${ }^{34}$ for measuring fatigue severity, the Numeric Pain Rating Scale (NPRS), ${ }^{35}$ the Questionnaire Version of the List of Threatening Experiences $(\mathrm{LTE}-\mathrm{Q})^{36}$ for social support and coping, the ConnorDavidson Resilience Scale (CD-RISC) ${ }^{37}$ for the ability to cope with stress, the Taiwanese Depression Questionnaire $(\mathrm{TDQ})^{38}$ for screening depression, and the Beck Anxiety Inventory (BAI) ${ }^{39}$ for the severity of anxiety, were used in the study. The BFI, NPRS, LTE-Q, CD-RISC, BAI, TDQ, and clinical and demographic data were collected by a trained research assistant.

\section{Statistical analyses}

Descriptive and inferential statistics were analysed using SPSS V.12.0 for Windows. Descriptive statistics $\left(\chi^{2}\right.$ and t-tests) were performed first to test the differences in demographic data and clinical characteristics between patients with and without depressive disorder. Logistic regression was used to test the factors associated with depressive disorder. Depression was set as dependent variable, and patients' characteristics were set as independent variables. We calculated both the adjusted OR and the 95\% CI. Structural equation modelling (SEM) was used to establish models of potential mechanisms underlying the linkage of associated factors and depression in patients with LC and HNC. The SEM statistical programme was analysed using SPSS Amos 24.0.

\section{RESULTS}

Data collection was completed for 113 HNC patients and 104 LC patients (table 1). LC patients were more often female $(46.2 \%$ vs $11.5 \%, \mathrm{p}<0.001)$, elderly $(61.3 \mathrm{y} / \mathrm{o}$ vs $52.6 \mathrm{y} / \mathrm{o}, \mathrm{p}<0.001)$, receiving targeted therapy $(40.6 \%$ vs $0 \%)$ and at an advanced stage $(84.0 \%$ vs $63.3 \%, \mathrm{p}<0.001)$ than HNC patients. HNC patients more often had a higher waist-to-hip ratio $(0.94$ vs $0.91, \mathrm{p}<0.05)$, and were more often alcohol users $(73.5 \%$ vs $26.0 \%, \mathrm{p}<0.001)$, tobacco smokers $(70.8 \%$ vs $41.3 \%, \mathrm{p}<0.001)$, betel nut users $(61.1 \%$ vs $16.3 \%, \mathrm{p}<0.001)$, undergoing an operation $(65.4 \%$ vs $22.8 \%)$ and receiving radiotherapy $(33.7 \%$ vs $11.9 \%)$. Notably, all 113 HNC patients were recruited from the inpatient ward. Twenty-three LC patients were recruited from the inpatient ward, and 81 were recruited from the outpatient department. The comparisons of demographic data and psychiatric morbidity are listed in online supplementary table 1 .

The psychiatric comorbidities at the pretreatment phase in patients are listed in table 2. The most common psychiatric diagnoses of the LC patients at the pretreatment phase were depressive disorders $(25.0 \%)$, followed by adjustment disorder (17.3\%), alcohol use disorder $(3.8 \%)$ and insomnia disorder (3.8\%). More HNC patients had alcohol use disorder $(49.6 \%$ vs $3.8 \%, \mathrm{p}<0.001)$ and a psychiatric diagnosis $(63.7 \%$ vs $50.0 \%, \mathrm{p}<0.05)$ compared with LC patients. More LC patients had depressive disorder $(25.0 \%$ vs $11.5 \%, \mathrm{p}<0.05)$ compared with $\mathrm{HNC}$ patients (table 2).

In the univariate analyses of the $113 \mathrm{HNC}$ patients (online supplementary table 2), factors significantly associated with depressive disorders included a self-harm history ( 15.4 vs $3.0 ; \mathrm{t}=4.17, \mathrm{p}<0.05$ ), being elderly ( 59.2 vs $51.7 ; \mathrm{t}=2.38, \mathrm{p}<0.05)$, having a lower educational level ( 6.9 vs $10.2 ; \mathrm{t}=-3.17, \mathrm{p}<0.05)$, severity of resilience $(26.7$ vs $31.4 ; \mathrm{t}=-2.50, \mathrm{p}<0.05)$ and severity of anxiety $(10.8$ vs $3.1 ; \mathrm{t}=2.62, \mathrm{p}<0.05)$. Using the stepwise forward model of logistic regression (table 3), we found that the independent risk factors for depression among HNC patients were a self-harm history ( $\mathrm{OR}=11.91 ; 95 \% \mathrm{CI}, 1.47$ to 96.83 ; $\mathrm{p}<0.05)$, and that a higher educational level $(\mathrm{OR}=0.77$; $95 \% \mathrm{CI}, 0.66$ to $0.91 ; \mathrm{p}<0.05)$ was negatively associated with depression.

Among the 104 LC patients (online supplementary table 3), factors significantly associated with depressive disorders included a self-harm history ( $15.4 \mathrm{vs} 1.3 ; \mathrm{t}=8.47$, $\mathrm{p}<0.05)$, severity of a stressor ( 1.4 vs $0.5 ; \mathrm{t}=4.93, \mathrm{p}<0.001)$, severity of lack of resilience (26.6 vs 31.6 ; $\mathrm{t}=-3.43$, $\mathrm{p}<0.05$ ), severity of fatigue $(3.8$ vs $2.5 ; \mathrm{t}=2.49, \mathrm{p}<0.05)$ and severity of anxiety ( 6.7 vs $3.4 ; \mathrm{t}=3.93, \mathrm{p}<0.001$ ). Using the stepwise forward model of logistic regression (table 3 ), severity of stressor $(\mathrm{OR}=2.78 ; 95 \% \mathrm{CI}, 1.50$ to 5.15 ; $\mathrm{p}<0.05)$ and severity of anxiety $(\mathrm{OR}=1.18 ; 95 \% \mathrm{CI}, 1.04$ to $1.34 ; \mathrm{p}<0.05)$ were determined as independent factors of depression among LC patients.

Using SEM, we found that both anxiety severity $(\beta=0.25$, $\mathrm{p}=0.004)$ and severity of stress $(\beta=0.36, p<0.001)$ were significantly linked with depressive disorder in patients with LC (figure 1A). Furthermore, we found that both years of education $(\beta=-0.31, \mathrm{p}<0.001)$ and a self-harm history $(\beta=0.22, p=0.012)$ were significantly linked with depressive disorder in patients with $\mathrm{HNC}$ (figure 1B).

\section{DISCUSSION}

To our knowledge, this is the first study comparing the prevalence and associated factors of depressive disorder between patients with HNC and those with LC. In our study, alcohol use disorder (27.6\%) was the most common psychiatric diagnosis, followed by adjustment disorder $(18.9 \%)$ and depressive disorder $(18.0 \%)$. Although alcohol use is common in patients with LC and HNC, in our study, alcohol use disorder was far more common in HNC patients than in LC patients.

LC patients were comorbid with depressive disorder more commonly than HNC patients (25\% vs $11.5 \%$ ). There were more female and elderly patients, more patients with an advanced stage and unemployed, and fewer users of tobacco, alcohol and betel nut among the LC patients than among the HNC patients. This 
Table 1 Demographic and clinical characteristics of patients with lung cancer, and head and neck cancer

\begin{tabular}{|c|c|c|c|c|c|}
\hline Characteristics & $\begin{array}{l}\text { Lung cancer } \\
\mathrm{N}(\%), \mathrm{n}=104\end{array}$ & $\begin{array}{l}\text { Head and neck cancer } \\
N(\%), n=113\end{array}$ & $\begin{array}{l}\text { Total } \\
\mathrm{N}(\%), \mathrm{n}=\mathbf{2 1 7}\end{array}$ & $\begin{array}{l}\chi^{2} / \\
\text { t-test }\end{array}$ & $P$ value \\
\hline Gender & & & & 32.17 & $<0.001$ \\
\hline Male & $56(53.80)$ & $100(88.50)$ & $156(71.89)$ & & \\
\hline Female & $48(46.20)$ & $13(11.50)$ & $61(28.11)$ & & \\
\hline Age, years mean (SD) & $61.26( \pm 10.30)$ & $52.58( \pm 10.81)$ & $56.74( \pm 11.40)$ & 6.04 & $<0.001$ \\
\hline Age demarcated & & & & 24.19 & $<0.001$ \\
\hline$\geq 50$ & $93(89.42)$ & $68(60.18)$ & $161(74.19)$ & & \\
\hline$<50$ & $11(10.58)$ & $45(39.82)$ & $56(25.81)$ & & \\
\hline Marital status & & & & 4.68 & $<0.05$ \\
\hline Unmarried & $19(18.27)$ & $35(30.97)$ & $54(24.88)$ & & \\
\hline Married & $85(81.73)$ & $78(69.03)$ & $163(75.12)$ & & \\
\hline Education, years mean (SD) & $9.91( \pm 4.20)$ & $9.80( \pm 3.71)$ & $9.85( \pm 3.94)$ & 0.22 & 0.83 \\
\hline Waist-to-hip ratio & $0.91( \pm 0.07)$ & $0.94( \pm 0.06)$ & $0.92( \pm 0.07)$ & 3.10 & $<0.05$ \\
\hline Unemployment & $73(70.19)$ & $55(48.67)$ & $128(58.99)$ & 10.37 & $<0.001$ \\
\hline Comorbid with other diseases & $50(48.08)$ & $50(44.25)$ & $100(46.08)$ & 0.32 & 0.57 \\
\hline Alcoholism & 27 (25.96) & $75(66.37)$ & $102(47.00)$ & 35.50 & $<0.001$ \\
\hline Smoking & $43(41.35)$ & $80(70.80)$ & $123(56.68)$ & 19.13 & $<0.001$ \\
\hline Betel nut & $17(16.35)$ & $69(61.06)$ & $86(39.63)$ & 45.26 & $<0.001$ \\
\hline Operation & $23(22.77)$ & 68 (65.38) & $91(44.39)$ & & \\
\hline Chemotherapy & $51(50.50)$ & $52(50.00)$ & $103(50.24)$ & & \\
\hline Radiotherapy & $12(11.88)$ & 35 (33.65) & 47 (22.93) & & \\
\hline Targeted drug & $42(41.58)$ & 0 & $42(20.49)$ & & \\
\hline Stage & & & & 10.99 & $<0.001$ \\
\hline Early & $16(16.00)$ & $36(36.73)$ & $52(26.26)$ & & \\
\hline Advanced & $84(84.00)$ & $62(63.27)$ & $146(73.74)$ & & \\
\hline NPRS, mean (range) & 1.81 (0 to 10$)$ & 1.50 (0 to 10$)$ & 1.65 (0 to 10$)$ & 1.06 & 0.29 \\
\hline TDQ, mean (SD) & $7.92( \pm 5.27)$ & $6.50( \pm 7.20)$ & $7.18( \pm 6.37)$ & 1.65 & 0.10 \\
\hline BAI, mean (SD) & $4.22( \pm 3.96)$ & $4.02( \pm 5.09)$ & $4.12( \pm 4.57)$ & 0.33 & 0.74 \\
\hline LTEQ, mean (range) & 0.74 (0 to 4$)$ & 0.84 (0 to 6$)$ & 0.79 (0 to 6$)$ & -0.76 & 0.45 \\
\hline CDRISC, mean (SD) & $30.35( \pm 6.80)$ & $30.86( \pm 6.53)$ & $30.61( \pm 6.66)$ & -0.57 & 0.57 \\
\hline BFI-S, mean (SD) & $2.82( \pm 2.29)$ & $2.78( \pm 2.59)$ & $2.80( \pm 2.44)$ & 0.13 & 0.90 \\
\hline BFI-I, mean (range) & $1.44(0$ to 0.85$)$ & 1.06 (0 to 10$)$ & 1.24 (0 to 10$)$ & 1.59 & 0.11 \\
\hline
\end{tabular}

BAI, Beck Anxiety Inventory; BFI-I, Brief Fatigue Inventory Interference subscale; BFI-S, Brief Fatigue Inventory Severity subscale; CD-RISC, Connor-Davidson Resilience Scale ; LTE-Q, Questionnaire Version of the List of Threatening Experiences ; NPRS, Numeric Pain Rating Scale; TDQ, Taiwanese Depression Questionnaire.

may partially explain why depressive disorder was more prevalent among patients with LC than among those with HNC; this finding was discussed in our previously published paper. ${ }^{40}$

Depressive disorder was the most frequent psychiatric diagnosis in LC patients. This result is compatible with that of previous studies, in which LC patients were commonly comorbid with depression, within a range of $11 \%$ to $44 \% .{ }^{20}$ A study from Thailand examined 104 LC patients using the MINI interview; 15 (14.4\%) of them were diagnosed as having MDD. ${ }^{41}$ In our study, 9.6\% of patients were diagnosed as having MDD, and $15.4 \%$ of patients had depressive disorder not otherwise specified. These two studies suggest that patients with LC are quite often comorbid with depressive disorder. In addition, depression morbidity in both studies was within the range of $14.4 \%$ to $25 \%$, which was lower than that in some studies of LC patients with a depression morbidity up to $44 \% .{ }^{20}$ The possible explanation is that both studies used a structured diagnostic interview by a clinician, which would render a lower morbidity rate than the self-rated questionnaires used in other clinical studies. Notwithstanding the lower morbidity rate of depression found when the clinician uses a structured diagnostic 
Table 2 Psychiatric diagnoses of patients with head and neck cancer and lung cancer

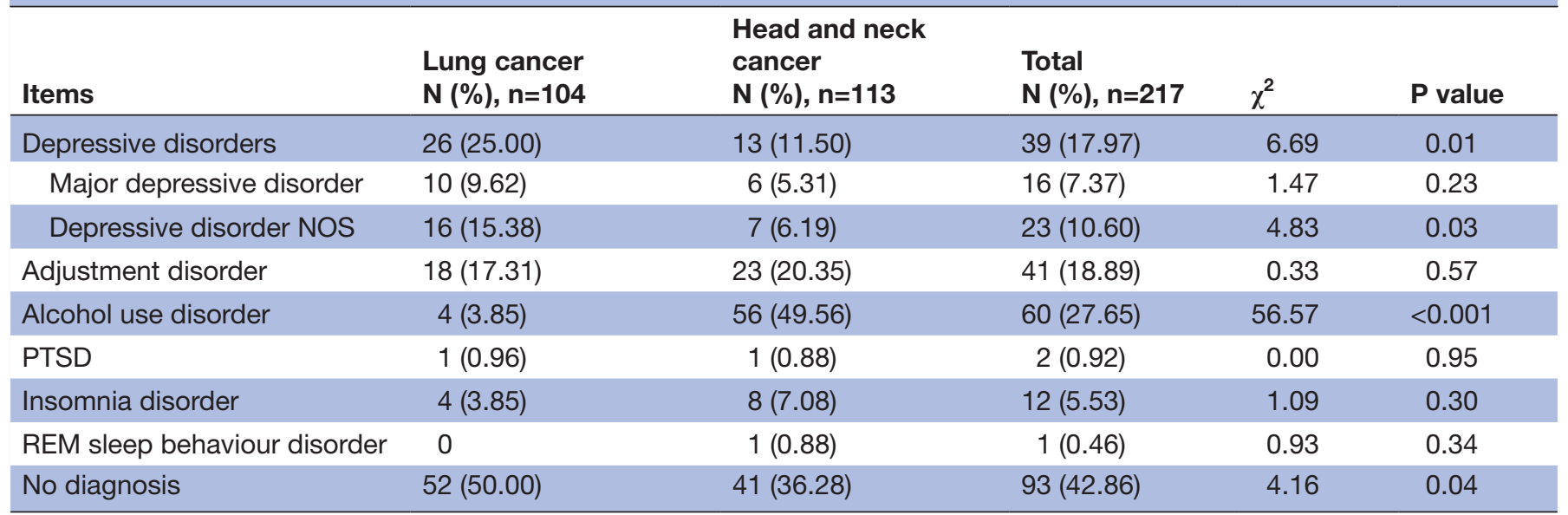

NOS, not otherwise specified; PTSD, Post-traumatic stress disorder; REM, rapid eye movement.

interview, the MINI or Structured Clinical Interview for DSM-5 Disorders (SCID-5) ${ }^{42}$ which are structured diagnostic interview instruments, are the gold standard for diagnosis, even beyond the psychiatric interview. Delayed diagnosis-related biological vulnerability and psychological impact can partially explain the high prevalence of depression in LC patients.

In our study, depressive disorder $(11.5 \%)$ was the third most common psychiatric diagnosis in HNC patients, following alcohol use disorder $(49.6 \%)$ and adjustment disorder $(20.4 \%)$. The morbidity of depressive disorder in our HNC patients was compatible with that of a metaanalysis study from Krebber et al, who reported that the prevalence of depression in HNC patients was $11 \%$ on the basis of diagnostic interviews, and $20 \%$ on the basis of self-reported questionnaires. ${ }^{43}$

The associated factors for depression in HNC patients were lower educational level and self-harm history, and in LC patients, the associated factors were severity of stressor and severity of anxiety. The possible explanations for the discrepancies in associated factors between HNC and LC patients are: (1) LC patients were at a more advanced stage than HNC patients when diagnosed, which contributed to the associated factors of severity of stressor and severity of anxiety; and (2) HNC patients included more male labourer patients, possibly with an impulsive personality, than LC patients, which contributed to the associated factors of lower educational level and self-harm history. To our knowledge, this is the first study to compare the associated factors of depression between LC and HNC patients. More prospective studies including different cancer types should be conducted to confirm these findings.

Prior studies showed that a high severity of suicide ideation in patients was associated with severity of depression and with having depressive disorder. ${ }^{44}$ Although very few studies have mentioned that patients with a self-harm history are at risk of developing depressive disorder, it can be speculated that patients with a self-harm history are vulnerable to stressors and tend to develop depressive disorder. HNC patients have a high morbidity of depression and a high risk of suicide. ${ }^{11}$ Up to now, few studies have found that HNC patients with a self-harm history are at risk of developing depression. Large-scale studies should be conducted to test this hypothesis, with the aim of preventing the co-occurrence of depression and suicide in $\mathrm{HNC}$ patients.

We found that lower educational level was one of the significant associated factors for depression among HNC patients. Our result was supported by those of previous studies, which have shown that a lower educational level correlated with depression in cancer patients. ${ }^{19}$ In studies focussing on depression in HNC patients, researchers found that patients with fewer than 9 or 12 years of education seemed to have more depression than patients with a college education. ${ }^{45}$

Table 3 Associated factors for depressive disorder at the pretreatment phase among patients with lung cancer and head and neck cancer: logistic regression analysis

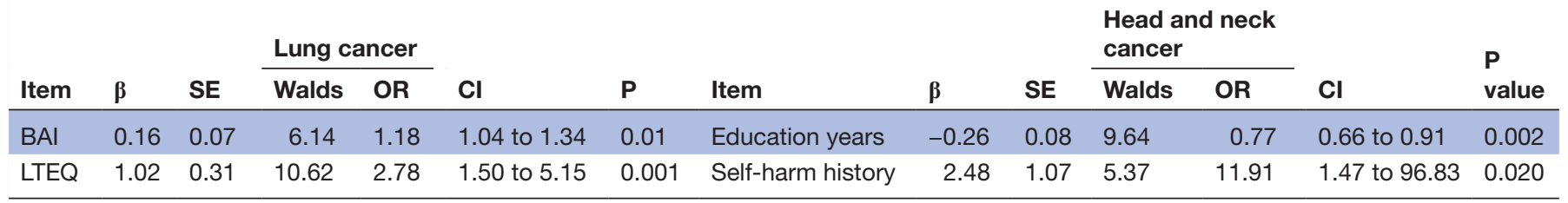

BAI, Beck Anxiety InventoryLTE-Q, Questionnaire Version of the List of Threatening Experiences . 
(A)

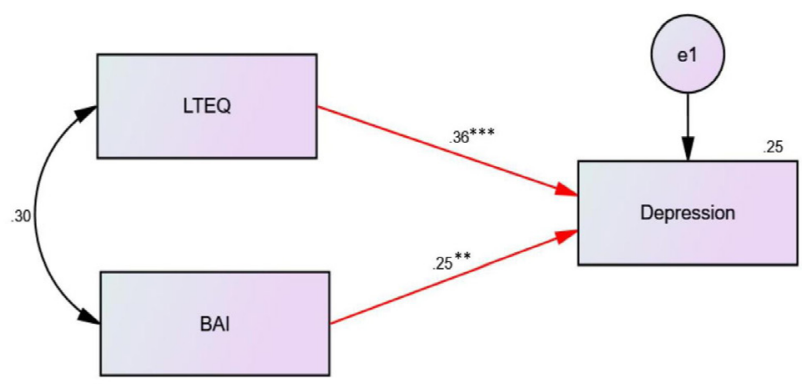

(B)

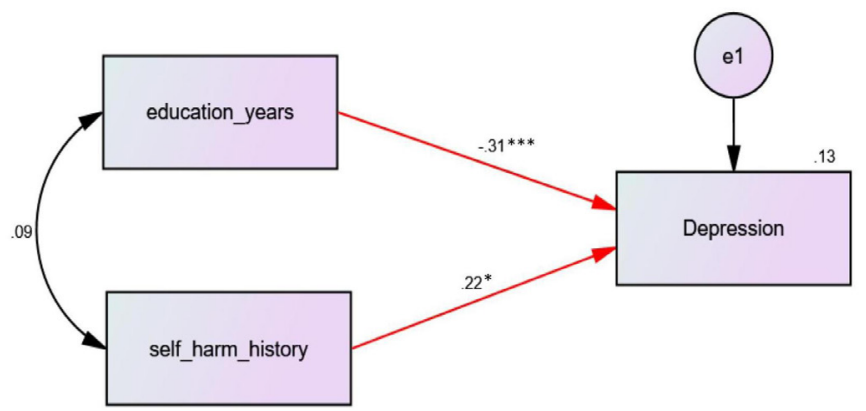

Figure 1 Structural equation modelling of factors linked to depressive disorder in patients with lung cancer $(A)$ and head and neck cancer (B). BAI, Beck Anxiety Inventory; LTE-Q, Questionnaire Version of the List of Threatening Experiences, ${ }^{*} p<0.05 ;{ }^{* *} p<0.01 ;{ }^{* *} p<0.001$

In this study, depressive cancer patients had a more than $18 \%$ severity of anxiety compared with non-depressive LC patients. Many patients with MDD suffer from subsyndromal anxiety symptoms or comorbid anxiety disorder. ${ }^{46}$ This comorbidity might support our finding that anxiety is an associated factor for depressive disorder. Possible explanations for why depressive cancer patients more commonly have anxiety symptoms are that cancer patients have to deal with the adverse effects of treatment, being abandoned, loss of self-control and even a survival crisis. ${ }^{47}$ To date, several studies have found that depression and anxiety are comorbid in cancer patients. ${ }^{14}$ However, very few studies have reported anxiety symptoms as an associated factor in cancer patients comorbid with depressive disorder. More studies are needed to confirm this finding.

Severity of stressor is the most robust associated factor for depressive disorder in LC patients. It is not unusual that a stressful life event is correlated with depression. ${ }^{48}$ However, only a few studies have detected a relationship between depressive disorder and severity of stressor in cancer patients. ${ }^{47}$ One study explored life events and depressive symptoms after breast cancer surgery, and found that a cancer-related traumatic stressor, financial problems and a tendency toward negativity may worsen depressive symptoms. ${ }^{49}$ The above-mentioned study can partially support our finding that a stressful life event is associated with depressive disorder in LC patients. Previous studies have shown that anxiety, fatigue or pain were associated with patients with HNC and LC. ${ }^{16} 5051$ The univariate analysis in our study revealed that fatigue and a lower level of resilience were correlated with depression in LC patients, and a lower level of resilience and older age were correlated with depression in HNC patients. However, these factors became non-significant when using a logistic regression analysis model. This result indicates that these factors might not independently affect depression in HNC patients or LC patients. SEM analysis showed that anxiety and stress might contribute to the development of depression in LC patients, whereas years of education and a self-harm history might contribute to the development of depression in HNC patients. It is very crucial that clinicians are aware of, and manage these contributing factors in LC and HLC patients so as to prevent these two groups from developing depression.

The high response rate and use of a structured clinical interview by the psychiatrists are two strengths of this study. Still, some limitations should be mentioned: (1) Our study design involved consecutive sampling, which may lead to sampling bias. However, a response rate of over $80 \%$ of the newly diagnosed cancer patients reduced the effect of this limitation; (2) our samples were from a general hospital, and may not be representative of the general population; and (3) this was a cross-sectional study, which does not allow investigation of the cancer patients' psychiatric disorder in the course of their cancer disease. So, further follow-up studies should be conducted to understand more precisely the depression morbidity of cancer patients and the risk factors involved.

\section{CONCLUSION}

We found that depression was more prevalent in LC patients than in HNC patients. Furthermore, the associated factors for depressive disorder in HNC patients were a self-harm history and lower educational level, and those in LC patients were severity of stressor and severity of anxiety. These findings give us a new insight into the importance of conducting intervention programmes directed toward aforementioned links to prevent these two patient groups from developing depression.

\section{Author affiliations}

${ }^{1}$ Department of Psychiatry, Kaohsiung Chang Gung Memorial Hospital and Chang Gung University College of Medicine, Kaohsiung, Taiwan

${ }^{2}$ Department of Otolaryngology, Kaohsiung Chang Gung Memorial Hospital and Chang Gung University College of Medicine, Kaohsiung, Taiwan

${ }^{3}$ Department of Internal Medicine, Kaohsiung Chang Gung Memorial Hospital and Chang Gung University College of Medicine, Kaohsiung, Taiwan

${ }^{4}$ Department of Cardiothoracic Vascular Surgery, Kaohsiung Chang Gung Memorial Hospital and Chang Gung University College of Medicine, Kaohsiung, Taiwan

${ }^{5}$ Department of Child and Adolescent Psychiatry, Kaohsiung Chang Gung Memorial Hospital and Chang Gung University College of Medicine, Kaohsiung, Taiwan

Acknowledgements The authors thank all participants in this study.

Contributors YL and C-FH participated in study design, executing study and drafting the manuscript. $\mathrm{YL}$ and $\mathrm{C}-\mathrm{FH}$ are co-first authors and contributed equally to this manuscript. C-YC, P-YL, M-CL, C-CW, H-IL, Y-CC and M-YC assisted with conception of the work, the acquisition, analysis and interpretation of data for the work. L-JW participated in study design, analysing and interpreting data and revised the manuscript. 
Funding This study was supported by a grant from Kaohsiung Chang Gung Memorial Hospital, Taiwan (CMRPG8E1381).

Competing interests None declared.

Patient and public involvement Patients and/or the public were not involved in the design, or conduct, or reporting, or dissemination plans of this research.

Patient consent for publication Not required.

Ethics approval Ethical approval was obtained from the Human Research Ethics Committee of Chang Gung Memorial Hospital (No. 106-0513C).

Provenance and peer review Not commissioned; externally peer reviewed.

Data availability statement Data are available upon reasonable request. Data are available upon reasonable request.

Open access This is an open access article distributed in accordance with the Creative Commons Attribution Non Commercial (CC BY-NC 4.0) license, which permits others to distribute, remix, adapt, build upon this work non-commercially, and license their derivative works on different terms, provided the original work is properly cited, appropriate credit is given, any changes made indicated, and the use is non-commercial. See: http://creativecommons.org/licenses/by-nc/4.0/.

ORCID iD

Liang-Jen Wang http://orcid.org/0000-0002-5320-1151

\section{REFERENCES}

1 Global Burden of Disease Cancer Collaboration, Fitzmaurice C, Allen $\mathrm{C}$, et al. Global, regional, and national cancer incidence, mortality, years of life lost, years lived with disability, and disability-adjusted life-years for 32 cancer groups, 1990 to 2015: a systematic analysis for the global burden of disease study. JAMA Oncol 2017;3:524-48.

2 Torre LA, Bray F, Siegel RL, et al. Global cancer statistics, 2012. CA Cancer J Clin 2015;65:87-108.

3 Siegel RL, Miller KD, Jemal A, et al. Cancer statistics, 2018. CA Cancer J Clin 2018;68:7-30.

4 Beynon RA, Lang S, Schimansky S, et al. Tobacco smoking and alcohol drinking at diagnosis of head and neck cancer and allcause mortality: results from head and neck 5000 , a prospective observational cohort of people with head and neck cancer. Int $J$ Cancer 2018;143:1114-27.

5 Marcus MW, Raji OY, Field JK. Lung cancer screening: identifying the high risk cohort. J Thorac Dis 2015;7:S156-62.

6 American Psychiatric Association. Diagnostic and statistical manual of mental disorders. 5 edn. Arlington, VA: American Psychiatric Publishing, 2013.

7 Liu Y, Wang J. Validity of the patient health questionnaire-9 for DSM-IV major depressive disorder in a sample of Canadian working population. J Affect Disord 2015;187:122-6.

8 Linden W, Vodermaier A, Mackenzie R, et al. Anxiety and depression after cancer diagnosis: prevalence rates by cancer type, gender, and age. J Affect Disord 2012;141:343-51.

9 Zimmaro LA, Sephton SE, Siwik CJ, et al. Depressive symptoms predict head and neck cancer survival: examining plausible behavioral and biological pathways. Cancer 2018;124:1053-60.

10 Laurence B, Mould-Millman N-K, Nero KE, et al. Depression and hospital admission in older patients with head and neck cancer: analysis of a national healthcare database. Gerodontology 2017;34:284-7.

11 Osazuwa-Peters N, Arnold LD, Loux TM, et al. Factors associated with increased risk of suicide among survivors of head and neck cancer: a population-based analysis. Oral Oncol 2018;81:29-34.

12 Satin JR, Linden W, Phillips MJ. Depression as a predictor of disease progression and mortality in cancer patients: a meta-analysis. Cancer 2009;115:5349-61.

13 Sullivan DR, Forsberg CW, Ganzini L, et al. Longitudinal changes in depression symptoms and survival among patients with lung cancer: a national cohort assessment. $J$ Clin Oncol 2016;34:3984-91.

14 Wu Y-S, Lin P-Y, Chien C-Y, et al. Anxiety and depression in patients with head and neck cancer: 6-month follow-up study. Neuropsychiatr Dis Treat 2016;12:1029.

15 van Beek FE, Jansen F, Mak L, et al. The course of symptoms of anxiety and depression from time of diagnosis up to 2 years followup in head and neck cancer patients treated with primary (chemo) radiation. Oral Oncol 2020;102:104576.

16 Korsten LHA, Jansen F, de Haan BJF, et al. Factors associated with depression over time in head and neck cancer patients: a systematic review. Psychooncology 2019;28:1159-83.
17 Rieke K, Schmid KK, Lydiatt W, et al. Depression and survival in head and neck cancer patients. Oral Oncol 2017;65:76-82.

18 Hopwood P, Stephens RJ, Party B. Depression in patients with lung cancer: prevalence and risk factors derived from quality-of-life data. J Clin Oncol 2000;18:893.

19 Haisfield-Wolfe ME, McGuire DB, Soeken K, et al. Prevalence and correlates of depression among patients with head and neck cancer: a systematic review of implications for research. Oncol Nurs Forum 2009;36:E104-25.

20 Massie MJ. Prevalence of depression in patients with cancer. J Natl Cancer Inst Monogr 2004:57-71.

21 Akechi T, letsugu T, Sukigara M, et al. Symptom indicator of severity of depression in cancer patients: a comparison of the DSM-IV criteria with alternative diagnostic criteria. Gen Hosp Psychiatry 2009;31:225-32.

22 Moubayed SP, Sampalis JS, Ayad T, et al. Predicting depression and quality of life among long-term head and neck cancer survivors. Otolaryngol Head Neck Surg 2015;152:91-7.

23 Akechi T, Okamura H, Nishiwaki Y, et al. Psychiatric disorders and associated and predictive factors in patients with unresectable nonsmall cell lung carcinoma: a longitudinal study. Cancer 2001;92:2609-22.

24 Uchitomi Y, Mikami I, Kugaya A, et al. Depression after successful treatment for nonsmall cell lung carcinoma. Cancer 2000;89:1172-9.

25 Schnoll RA, Malstrom M, James C, et al. Correlates of tobacco use among smokers and recent quitters diagnosed with cancer. Patient Educ Couns 2002;46:137-45.

26 Okuyama T, Tanaka K, Akechi T, et al. Fatigue in ambulatory patients with advanced lung cancer: prevalence, correlated factors, and screening. J Pain Symptom Manage 2001;22:554-64.

27 Lo C, Zimmermann C, Rydall A, et al. Longitudinal study of depressive symptoms in patients with metastatic gastrointestinal and lung cancer. J Clin Oncol 2010;28:3084-9.

28 Shi Y, Gu F, Hou L-L, LI H, et al. Self-reported depression among patients with non-small cell lung cancer. Thorac Cancer 2015;6:334-7.

29 Agarwal M, Hamilton JB, Moore CE, et al. Predictors of depression among older African American cancer patients. Cancer Nurs 2010;33:156-63.

30 Uchitomi Y, Mikami I, Nagai K, et al. Depression and psychological distress in patients during the year after curative resection of nonsmall-cell lung cancer. J Clin Oncol 2003;21:69-77.

31 Health Promotion Administration. Taiwan cancer registry, 2018.

32 Cunningham JB. Power, effect and sample size using GPower: practical issues for researchers and members of research ethics committees. Evidence Based Midwifery 2007;5:132-6.

33 Sheehan DV, Lecrubier Y, Sheehan KH, et al. The Mini-international neuropsychiatric interview (M.I.N.I.): the development and validation of a structured diagnostic psychiatric interview for DSM-IV and ICD10. J Clin Psychiatry 1998;59:22-33.

34 Mendoza TR, Wang XS, Cleeland CS, et al. The rapid assessment of fatigue severity in cancer patients: use of the brief fatigue inventory. Cancer 1999;85:1186-96.

35 Jensen MP. The validity and reliability of pain measures in adults with cancer. J Pain 2003;4:2-21.

36 Brugha $\mathrm{T}$, Bebbington $\mathrm{P}$, Tennant $\mathrm{C}$, et al. The list of threatening experiences: a subset of 12 life event categories with considerable long-term contextual threat. Psychol Med 1985;15:189-94.

37 Connor KM, Davidson JRT. Development of a new resilience scale: the Connor-Davidson resilience scale (CD-RISC). Depress Anxiety 2003;18:76-82.

38 Lee $\mathrm{Y}$, Yang MJ, Lai TJ, et al. Development of the Taiwanese depression questionnaire. Chang Gung Med J 2000;23:688-94.

39 Beck AT, Epstein N, Brown G, et al. An inventory for measuring clinical anxiety: psychometric properties. J Consult Clin Psychol 1988;56:893-7.

40 Lee Y, Chien C-Y, Lin P-Y, et al. Psychiatric morbidity, pain, anxiety and depression in patients with head and neck cancer and lung cancer. Int J Head Neck Science 2017;1:191-8.

41 Maneeton B, Maneeton N, Reungyos J, et al. Prevalence and relationship between major depressive disorder and lung cancer: a cross-sectional study. Onco Targets Ther 2014;7:815-21.

42 First MB, Williams JBW, Karg RS, et al. Structured clinical interview for DSM-5 disorders (Scid-5-cv) - clinician version. 1 edn. Arlington, VA: American Psychiatric Publishing, 2015.

43 Krebber AMH, Buffart LM, Kleijn G, et al. Prevalence of depression in cancer patients: a meta-analysis of diagnostic interviews and selfreport instruments. Psychooncology 2014;23:121-30.

44 O'Brien G, Holton AR, Hurren K, et al. Deliberate self harm-correlates of suicidal intent and severity of depression. Acta Psychiatr Scand 1987;75:474-7. 
45 Duffy SA, Ronis DL, Valenstein M, et al. Depressive symptoms, smoking, drinking, and quality of life among head and neck cancer patients. Psychosomatics 2007;48:142-8.

46 Zimmerman M, McDermut W, Mattia Jl. Frequency of anxiety disorders in psychiatric outpatients with major depressive disorder. Am J Psychiatry 2000;157:1337-40.

47 Brown Johnson CG, Brodsky JL, Cataldo JK, et al. Lung cancer stigma, anxiety, depression, and quality of life. J Psychosoc Oncol 2014;32:59-73.

48 Finlay-Jones R, Brown GW. Types of stressful life event and the onset of anxiety and depressive disorders. Psychol Med 1981;11:803-15.
49 Golden-Kreutz DM, Andersen BL. Depressive symptoms after breast cancer surgery: relationships with global, cancer-related, and life event stress. Psychooncology 2004;13:211-20.

50 Hung M-S, Chen I-C, Lee C-P, et al. Incidence and risk factors of depression after diagnosis of lung cancer: a nationwide populationbased study. Medicine 2017;96:e6864.

51 Lee Y, Lin P-Y, Lin M-C, et al. Morbidity and associated factors of depressive disorder in patients with lung cancer. Cancer Manag Res 2019;11:7587-96. 